\title{
Mader Tools
}

\section{Frank Göring ${ }^{1 \dagger}$}

${ }^{1}$ Fakultät für Mathematik, Technische Universität Chemnitz, D-09107 Chemnitz.

email: frank.goering@mathematik.tu-chemnitz.de

The deep theorem of Mader concerning the number of internally disjoint $H$-paths is a very powerfull tool. Nevertheless its use is very difficult, because one has to deal with a very reach family of separators. This paper shows several ways to strengthen Mader's theorem by certain additional restrictions of the appearing separators.

Keywords: graph, $H$-path, separator

\section{Preliminaries and Results}

For notations not defined here we refer to (1). Unless otherwise stated, $k$ is an arbitrary integer, $G$ is an arbitrary finite simple graph (loops and multiple edges are forbidden), $U$ is an arbitrary subgraph of $G$, $X$ and $H$ are arbitrary disjoint subsets of $V(G)$ and $Y$ is an arbitrary subset of $E(G-X-H)$. A path having exactly its endvertices in $H$ is called an $H$-path. The maximum number of independent $H$-paths we denote by $p_{G}(H)$. [Y] denotes the graph with edge set $Y$ whose vertex set is the set of all vertices incident with at least one edge of $Y$. Let $\mathcal{C}(G)$ denote the set of components of $G$ and $\partial_{G}(U)$ denote the set of vertices of $U$ incident with at least one edge of $G-E(U)$. A pair $(X, Y)$ is called $H$-separator of $G$, if each $H$-path of $G$ contains a vertex of $X$ or an edge of $Y$. Let $\mathcal{S}$ be the set of all $H$-separators of $G-E(G[H])$. A vertex $x^{\prime}$ of $G$ is called big brother of a vertex $x$ of $G$, if the neighborhood of $x^{\prime}$ in $G$ contains the neighborhood of $x$ in $G-x^{\prime}$.

According to (1) we define the permeability of a pair $(X, Y)$ by:

$$
\mathbf{M}_{G}(X, Y)=|X|+\sum_{C \in \mathcal{C}([Y])}\left\lfloor\frac{\left|\partial_{G-X}(C)\right|}{2}\right\rfloor
$$

Mader's Theorem (cf. (2)) can be rewritten as follows (cf. (1).)

\section{Theorem 1 (Mader, 1978)}

$$
p_{G}(H)=|E(G[H])|+\min \left\{M_{G}(X, Y) \mid(X, Y) \in \mathcal{S}\right\}
$$

\footnotetext{
${ }^{\dagger}$ Research supported by the "Mathematics in Information Society" project carried out by Alfréd Rényi Institute of Mathematics Hungarian Academy of Sciences, in the framework of the European Community's "Confirming the International Role of Community Research" programme.
}

1365-8050 (c) 2005 Discrete Mathematics and Theoretical Computer Science (DMTCS), Nancy, France 
Note, that here $H$ is a set of vertices. To get this from the version of Mader's theorem in (1), you have to apply the version of (1) with the graph $G[H]$ instead of $H$.

Let a subset $\mathcal{S}^{\prime}$ of $\mathcal{S}$ be a Mader-Set, whenever Theorem 1 remains valid if $S$ is replaced by $S^{\prime}$. In other words, a subset $\mathcal{S}^{\prime}$ of $\mathcal{S}$ is a Mader-Set, iff for each element $(X, Y)$ of $\mathcal{S}$ there is an element $\left(X^{\prime}, Y^{\prime}\right)$ of $\mathcal{S}^{\prime}$ with $\mathrm{M}_{G}\left(X^{\prime}, Y^{\prime}\right) \leq \mathrm{M}_{G}(X, Y)$. Note that a subset of $\mathcal{S}$ containing a Mader-Set is a Mader-Set, too.

The following conditions for elements $(X, Y)$ of $\mathcal{S}$ will be discussed:

- Odd Border Condition $(O B)$

For each component $C$ of $[Y]$ the number $\left|\partial_{G-X} C\right|$ is odd.

- Big Brother Vertex Condition (BV): If $x \in X$ and $x^{\prime}$ is a big brother of $x$, then $x^{\prime} \in X$.

- Symmetric Edge Condition (SE): If $v$ and $v^{\prime}$ are two vertices of $G-H-X$ such that the neighborhood of $v^{\prime}$ in $G-v$ equals the neighborhood of $v$ in $G-v^{\prime}$, then the neighborhood of $v^{\prime}$ in $[Y]-v$ equals the neighborhood of $v$ in $[Y]-v^{\prime}$.

- Edge Component Condition (EC): For each edge $e$ of $G-H-X-Y$ and each component $C$ of $[Y] \cup(V(G-H-X), \emptyset)$ there is a path $P$ in $G-X-Y-C$ containing an element of $H$ and an endvertex of $e$.

- Half Border Condition (HB): For each $C \in[Y]$ and each $B \subseteq \partial_{G-X} C$ with $2|B| \geq\left|\partial_{G-X} C\right|$ there are two vertexdisjoint $H B$-paths in $G-X$.

For a subset $Q$ of the set of conditions $\{\mathrm{OB}, \mathrm{BV}, \mathrm{BE}, \mathrm{EC}\}$ let $\mathcal{S}(Q)$ be the subset of $\mathcal{S}$ satisfying all conditions in $Q$. Our main results are as follows:

Theorem $2 \mathcal{S}(\{O B, S E, H B, E C\})$ is a Mader-Set.

Theorem $3 \mathcal{S}(\{B V, S E, H B, E C\})$ is a Mader-Set.

Theorem 4 There is a graph $G$ and a subset $H$ of $V(G)$ such that $\mathcal{S}(\{O B, B V\})$ is not a Mader-Set.

In other words, Theorem 2 and Theorem 3 state, that for each graph $G$ and each subset $H$ of $V(G)$ the set $\mathcal{S}^{*}(G, H)$ of $H$-separators of $G$ with minimal permeability has (possibly equal) elements $\left(X_{1}, Y_{1}\right)$ and $\left(X_{2}, Y_{2}\right)$ such that $\left(X_{1}, Y_{1}\right)$ satisfies the Odd Border Condition, the Symmetric Edge Condition, the Half Border Condition and the Edge Component Condition, and $\left(X_{2}, Y_{2}\right)$ satisfies the Big Brother

Vertex Condition, the Symmetric Edge Condition, the Half Border Condition and the Edge Component Condition.

Theorem 4 states, that there is a graph $G$ and a subset $H$ of $V(G)$, such that none of the elements of $\mathcal{S}^{*}(G, H)$ satisfies the Odd Border Condition and the Big Brother Vertex Condition.

\section{Motivation}

Why dealing with such mysterious conditions? The Odd Border Condition helps to simplify the formula for the permeability of an $H$ separator:

Theorem 5 Let $G$ be a graph, $H$ be a subset of $G$, and $(X, Y)$ be an $H$-separator of $G$ satisfying the Odd Border Condition. Then for the permeability of $(X, Y)$ the following equation holds:

$$
M_{G}(X, Y)=|X|+\frac{\left|\partial_{G-X}[Y]\right|-|\mathcal{C}([Y])|}{2}
$$


In order to motivate the remaining three conditions, we regard an application of Mader's Theorem: Suppose, a function $f$ mapping $H$ into the set of nonnegative integers is given. We are interested in a 'separator-like' condition for the existence of a set of $p$ independent $H$-paths such that in the graph $U$ being the union of all this paths $f(h) \geq d_{U}(h)$ holds for all $h \in H$. Such a problem appears for instance, if one wants to prove the $f$-factor theorem with help of Mader's Theorem.

Let the graph $R(G, f)$ be obtained from $G$ by the following procedure: Let $G^{\prime}$ be the graph obtained from $G$ by intersecting each edge $e$ of $G[H]$ by a vertex $h_{e}$. In $G^{\prime}$ sequentially replace each vertex $v$ of $H$ by a complete bypartite graph $R_{v}$ whose partition classes $A_{v}$ and $B_{v}$ satisfy $\left|A_{v}\right|=d_{G}(v)+1$ and $\left|B_{v}\right|=f(v)$. In each step each edge incident with $v$ (say $(u, v)$ ) of $G^{\prime}$ has to be replaced by an edge $(u, a)$ with $a \in A_{v}$ such that in the resulting graph $R(G, f)$ only one vertex $a_{v}$ of $A_{v}$ has all its neighbors in $B_{v}$. We call $R(G, f)$ the $f$-replacement of $G$.

The set $H_{R}(G, f)=\left\{a_{v} \mid v \in H\right\}$ we call $f$-replacement of $H$ in $G$. With this definitions we find the following lemma:

Lemma $6 G$ has a set of $p$ independent $H$-paths such that each vertex $v$ of $H$ is contained in at most $f(v)$ of this paths if and only if $R(G, f)$ has a set of $p$ independent $H_{R}(G, f)$-paths.

Using Mader's Theorem for $R(G, f)$ instead of $G$ and $H_{R}(G, f)$ instead of $H$ we get

Lemma $7 G$ has a set of $p$ independent $H$-paths such that each vertex $v$ of $H$ is contained in at most $f(v)$ of this paths if and only if each $H_{R}(G, f)$-separator $(X, Y)$ of $R(G, f)$ satisfies $p<p(X, Y)$.

Now, we are nearly done. We have to retranslate this condition to a condition for the graph $G$, the set $H$, and the function $f$ only. To reconstruct $G$ from $R(G, f)$, for each $v \in H$ we have to contract the graph $R_{v}$ to the vertex $v$, and after that for each $e \in E(G[H])$ we have to delete $h_{e}$ and to add $e$. But, without any knowledge about a special structure of $H_{R}(G, f)$-separators in $R(G, f)$, we loose too much information by doing the contractions.

The situation changes rapidly, if we first apply Theorem 3 with $R(G, f)$ instead of $G$ and $H_{R}(G, f)$ instead of $H$. Using this we prove the following Lemma:

Lemma $8 G$ has a set of $p$ independent $H$-paths such that each vertex $v$ of $H$ is contained in at most $f(v)$ of this paths if and only if each $H_{R}(G, f)$-separator $(X, Y)$ of $R(G, f)$ that satisfies the following conditions also satisfies $p<p(X, Y)$.

Here are the conditions:

For each element $v$ of $H$ one of the following statements holds:

1. $V\left(R_{v}\right) \cap X=B_{v}$ and no edge of $Y$ is incident with $A_{v}$,

2. $V\left(R_{v}\right) \cap X=\emptyset$ and $Y$ contains each edge of $R(G, f)$ incident with $A_{v} \backslash\left\{a_{v}\right\}$.

3. $V\left(R_{v}\right) \cap X=\emptyset$ and no edge of $Y$ is incident with $A_{v}$.

For each edge e of $G[H]$ we have $h_{e} \in X$ if and only if for each edge $v$ incident with $e$ the third statement $\left(V\left(R_{v}\right) \cap X=\emptyset\right.$ and no edge of $Y$ is incident with $\left.A_{v}\right)$ holds.

By Lemma 8, it is possible to interpret the resulting structure in $G$, directly. For this, let a pair $(X, Y)$ be $(G, H)$-valid, if $G-X-Y$ has no $H$-path and $\partial_{G-X}[Y]$ is disjoint to $H$.

We derive the following Theorem: 
Theorem 9 Given a graph $G$, a subset $H$ of its vertex set, and a function $f$ that maps $H$ to the set of non-negative integers.

The maximum number of independent $H$-paths, for which each vertex $v$ of $H$ is contained in at most $f(v)$ of this paths, equals the minimum of

$$
|E(G[H \backslash(X \cup V([Y]))])|+|X \backslash H|+\sum_{x \in H \cap X} f(x)+\sum_{C \in \mathcal{C}([Y])}\left\lfloor\frac{1}{2}\left(\left|\partial_{G-X} C\right|+\sum_{v \in H \cap V(C)} f(v)\right)\right\rfloor
$$

taken over all $(G, H)$-valid pairs $(X, Y)$.

\section{References}

[1] R. Diestel, Graph Theory, Springer, Graduate Texts in Mathematics 173(2000).

[2] W. Mader, Ueber die Maximalzahl kreuzungsfreier H-Wege, Arch. Math. 31 (1978), pp 387-402.

[3] K. Menger, Zur allgemeinen Kurventheorie, Fund. Math. 10 (1927) 96-115. 